\title{
FULL GROUPS AND SOFICITY
}

\author{
GÁBOR ELEK
}

(Communicated by Varghese Mathai)

\begin{abstract}
First, we answer a question of Giordano and Pestov by proving that the full group of a sofic equivalence relation is a sofic group. Then, we give a short proof of the theorem of Grigorchuk and Medynets that the topological full group of a minimal Cantor homeomorphism is LEF. Finally, we show that for certain non-amenable groups all the generalized lamplighter groups are sofic.
\end{abstract}

\section{INTRODUCTION}

1.1. Sofic groups and LEF groups. The notion of sofic groups was introduced by Weiss [12] and Gromov [5] (in a somewhat different form). A group $\Gamma$ is sofic if for any finite set $F \subset \Gamma$ and $\epsilon>0$ there exists a finite set $A$ and a mapping $\Theta: \Gamma \rightarrow \operatorname{Map}(A)$ such that $([3])$

- If $f, g, f g \in F$, then $d_{H}(\Theta(f g)-\Theta(f) \Theta(g)) \leq \epsilon$, where

$$
d_{H}(\alpha, \beta)=\frac{|\{x \in A \mid \alpha(x) \neq \beta(x)\}|}{|A|} .
$$

- If $1 \neq f \in F$, then $d_{H}(\Theta(f), 1)>1-\epsilon$.

- $\Theta(1)=1$.

All amenable and residually finite groups are sofic. It is an open question whether non-sofic groups exist. If we add the extra requirement that $\Theta(f g)=\Theta(f) \Theta(g)$, then we get the class of LEF-groups (locally embeddable into finite groups). This class of groups was introduced by Gordon and Vershik [11. Clearly, all residually finite groups are LEF. However, simple, finitely presented groups are not LEF. Nevertheless, by a recent result of Juschenko and Monod [6] (and Theorem 2), there exist simple, finitely generated LEF-groups.

1.2. Sofic equivalence relations. Let $X=\{0,1\}^{\mathbf{N}}$ be the standard Borel space with the natural product measure $\mu$. Let $\Phi: \mathbf{F}_{\infty} \curvearrowright X$ be a (not necessarily free) Borel action of the free group of countably infinite generators $\left\{\gamma_{1}, \gamma_{1}^{-1}, \gamma_{2}, \gamma_{2}^{-1}, \ldots\right\}$ preserving $\mu$. Note that $\mathbf{F}_{\infty}=\bigcup_{r=1}^{\infty} \mathbf{F}_{r}$, where $\mathbf{F}_{r}$ is the free group of rank $r$. Hence, we also have probability measure preserving (p.m.p.) Borel actions $\Phi_{r}: \mathbf{F}_{r} \curvearrowright X$. We say that $x, y \in X$ are equivalent, $x \sim_{\Phi} y$ if there exists $w \in \mathbf{F}_{\infty}$, such that $w(x)=y$. Note that slightly abusing the notation we write $w(x)$ instead of $\Phi(w)(x)$. Thus, the action $\Phi$ represents a countable measured equivalence relation $E_{\Phi}$ on $X$. Similarly, each $\Phi_{r}$ represents a countable measured equivalence relation $E_{\Phi_{r}}$ on $X$,

Received by the editors November 18, 2012 and, in revised form, November 6, 2013.

2010 Mathematics Subject Classification. Primary 20F65, 37A20.

This work was supported in part by a Marie Curie grant, TAMOP 4.2.1/B-09/1/KMR-2010003 and MTA Renyi "Lendulet" Groups and Graphs Research Group. 
and $E_{\Phi}=\bigcup_{i=1}^{\infty} E_{\Phi_{r}}$. Each equivalence relation $E_{\Phi_{r}}$ defines a graphing [7] $G_{r}$ on $X$ :

- $V\left(G_{r}\right)=X$.

- $(x, y) \in E\left(G_{r}\right)$ if $\gamma_{i} x=y$ or $\gamma_{i} y=x$ for some $i$ (so, there may be loops in $\left.G_{r}\right)$.

Observe that each component of $G_{r}$ is a countable graph of bounded vertex degrees. We label each directed edge $(x, y)$ with all the generators mapping $x$ to $y$. Thus an edge, even a loop, may have multiple labels.

Now let us consider transitive actions of $\mathbf{F}_{r}$ on countable sets. If $\alpha: \mathbf{F}_{r} \curvearrowright Y$ is such an action, then we have a bounded degree graph structure on $Y$ with multiple labels on the edges from the set $\left\{\gamma_{1}, \gamma_{1}^{-1}, \ldots, \gamma_{r}, \gamma_{r}^{-1}\right\}$. Let $T_{r}$ be the set of graphs of all countable $\mathbf{F}_{r}$-actions with a distinguished vertex (the root) such that all the vertices are labeled by the elements of $\{0,1\}^{r}$. Let $G \in T_{r}$. We define the the $k$-ball around the root $x, B_{k}(x)$, as the induced subgraph on vertices of $G$ in the form of $w(x)$, where $w \in \mathbf{F}_{r}$ is a reduced word of length at most $k$. That is, $B_{k}(x)$ is the ball centered at $x$ of radius $k$ with respect to the shortest path metric of $G$. The ball $B_{k}(x)$ is a finite rooted graph with edge-colors from the set $\left\{\gamma_{1}, \gamma_{1}^{-1}, \ldots, \gamma_{r}, \gamma_{r}^{-1}\right\}$ and vertex labels from the set $\{0,1\}^{r}$. We denote the set of all possible $k$-balls arising from $\mathbf{F}_{r}$-actions by $U_{r}^{k}$. We can define a compact metric structure on the set $T_{r}$ the following way. Let $d_{r}(G, H)=\frac{1}{2^{k}}$ if $k$ is the maximal number such that the $k$-balls around the roots of $G$, resp. $H$, are isomorphic as rooted, labeled graphs.

Observe that if $\Theta: \mathbf{F}_{\infty} \curvearrowright X$ is a p.m.p. action, then for each $r \geq 1$ and $x \in X$ one can associate an element $G(\Theta, x) \in T_{r}$. Namely, the orbit graph of $x$, where the vertex labels are given by the $X$-values, restricted on the first $r$ coordinates. Thus, we have a Borel map $\pi_{\Theta}: X \rightarrow T_{r}$. For $\kappa \in U_{r}^{k}$, let $\mu_{\Theta_{r}}^{k}(\kappa)=\left(\pi_{\theta}\right)_{\star}(\mu)\left(L_{\kappa}\right)$, where $L_{\kappa} \subset T_{r}$ is the set of elements $G$ such that the $k$-ball around the root of $G$ is isomorphic to $\kappa$. In other words, $\mu_{\Theta_{r}}^{k}(\kappa)$ is the probability that the $k$-ball around a $\mu$-random element of $X$ is isomorphic to $\kappa$. Now let $\alpha: \mathbf{F}_{r} \curvearrowright Y$ be an $\mathbf{F}_{r}$-action on a finite set. Then for each element $y$ of $Y$, we can associate an element of $T_{r}$; namely, $Y$ itself with root $y$. Hence, we can define a probability distribution $\mu_{\alpha}^{k, r}$ on $U_{r}^{k}$. Following [1] we say that the action $\Theta: \mathbf{F}_{\infty} \curvearrowright X$ is sofic if for all $r \geq 1$, there exists a sequence of finite $\mathbf{F}_{r}$-actions $\left\{\alpha_{n}\right\}_{n=1}^{\infty}$ such that for each $k \geq 1$ and $\kappa \in U_{r}^{k}$,

$$
\lim _{n \rightarrow \infty} \mu_{\alpha_{n}}^{k, r}(\kappa)=\mu_{\Theta_{r}}^{k}(\kappa) .
$$

In [1] the authors proved that

- Soficity is a property of the underlying equivalence relations. That is, if an action $\Theta_{1}$ is orbit equivalent to a sofic action $\Theta_{2}$, then $\Theta_{2}$ is sofic as well.

- Treeable equivalence relations are sofic.

- Actions associated to Bernoulli shifts of sofic groups are sofic.

1.3. Full groups. Let $E(X, \mu)$ be a countable, measured equivalence relation on a Borel set $X$ with invariant measure $\mu$. The Borel full group of $E$ is the group $[E]_{B}$ of all Borel bijections $T: X \rightarrow X$ such that for any $x \in X, T(x) \sim_{E} x$. We call two such bijections $T_{1}, T_{2}$ equivalent if

$$
\mu\left(\left\{x \in X \mid T_{1}(x)=T_{2}(x)\right\}\right)=1 .
$$


The measurable full group $[E]$ is the group formed by the equivalence classes. Obviously, $[E]=[E]_{B} / N$, where $N$ is the normal subgroup of elements in $[E]_{B}$ fixing almost all points of $X$.

Now, let $T: C \rightarrow C$ be a homeomorphism of the Cantor set $C$. The topological full group [[T]] is the group of homeomorphisms $S: C \rightarrow C$ such that $C$ can be partitioned into finitely many clopen sets $C=\bigcup_{i=1}^{n} A_{i}$ such that $S_{\mid A_{i}}=T^{n_{i}}$ for some integer $n_{i}$.

1.4. Results. Answering a question of Giordano and Pestov 1 we prove the following theorem.

Theorem 1. The measurable full group of a sofic equivalence relation is sofic.

Then, we give a very short proof of a result of Grigorchuk and Medynets [4].

Theorem 2. The topological full group of a minimal Cantor homeomorphism is $L E F$.

Let $X$ be a countably infinite set and $\Gamma$ be a countable group acting faithfully and transitively on $X$. Then $\Gamma$ can be represented by automorphisms on the Abelian group $\bigoplus_{x \in X}\{0,1\}$. The groups $\bigoplus_{x \in X}\{0,1\} \rtimes \Gamma$ are called the lamplighter group of the $\Gamma$-action. If the action is the natural translation action on $\Gamma$, then we get the classical lamplighter group of $\Gamma$. Paunescu [10] proved that if $\Gamma$ is sofic, then the classical lamplighter group $\bigoplus_{\gamma \in \Gamma}\{0,1\} \rtimes \Gamma$ is sofic. If $\Gamma$ is amenable, then all its generalized lamplighter groups are amenable, hence sofic. Nevertheless, we show that there exist non-amenable groups for which all the generalized lamplighter groups are sofic.

Theorem 3. Let $\Gamma^{k}$ be the $k$-fold free product of the cyclic group of two elements. Then, for any transitive, faithful action of $\Gamma^{k}$ on a countable set the associated lamplighter group is LEF.

\section{Compressed sofic Representations}

Let $\Gamma$ be a countable sofic group with elements $\left\{\gamma_{1}, \gamma_{2}, \ldots\right\}$. A compressed sofic representation of $\Gamma$ is defined in the following way. For any $i \geq 1$ we have a constant $\epsilon_{i}>0$, and for any $n \geq 1$ we have mappings $\Theta_{n}: \Gamma \rightarrow \operatorname{Map}\left(A_{n}\right)$ such that $\left|A_{n}\right|<\infty$ satisfying the following condition: For all $r>0$ and $\epsilon>0$ there exists $K_{r, \epsilon}>0$ such that if $n>K_{r, \epsilon}$, then

- $d_{H}\left(\Theta_{n}\left(\gamma_{i} \gamma_{j}\right) \Theta_{n}\left(\gamma_{i}\right) \Theta_{n}\left(\gamma_{j}\right)\right)<\epsilon$ if $1 \leq i, j \leq r$.

- $d_{H}\left(\Theta_{n}\left(\gamma_{i}\right), I d\right)>\epsilon_{i}$ if $1 \leq i \leq r$.

Thus, in a compressed sofic representation we allow a large amount of fixed points for each $\gamma \in \Gamma$.

Lemma 2.1. If $\Gamma$ has a compressed sofic representation, then $\Gamma$ is sofic.

Proof. Let $\tilde{\Theta}_{n}^{k}: \Gamma \rightarrow \operatorname{Map}\left(A_{n}^{k}\right)$ be defined by

$$
\tilde{\Theta}_{n}^{k}(\gamma)\left(x_{1}, x_{2}, \ldots, x_{k}\right)=\left(\Theta_{n}(\gamma)\left(x_{1}\right), \Theta_{n}(\gamma)\left(x_{2}\right), \ldots\right) .
$$

Observe that if $\gamma, \delta \in \Gamma$, then

- $d_{H}\left(\tilde{\Theta}_{n}^{k}(\gamma \delta), \tilde{\Theta}_{n}^{k}(\gamma) \tilde{\Theta}_{n}^{k}(\delta)\right) \leq\left(1-d_{H}\left(\Theta_{n}(\gamma \delta), \Theta_{n}(\gamma) \Theta_{n}(\delta)\right)\right)^{k}$.

- $d_{H}\left(\tilde{\Theta}_{n}^{k}(\gamma), I d\right)>1-\left(1-d_{H}\left(\Theta_{n}(\gamma), I d\right)\right)^{k}$.

\footnotetext{
${ }^{1}$ MR2566316-MathSciNet Review.
} 
Hence, we can choose $\epsilon, n$ and $k$ appropriately to obtain for any $F \subset \Gamma$ and $\epsilon^{\prime}>0$ a map $\Theta$ as in the Introduction, proving the soficity of $\Gamma$.

\section{The Proof of Theorem 1}

Let $\Phi: \mathbf{F}_{\infty} \curvearrowright\{0,1\}^{\mathbf{N}}$ be a sofic action preserving the product measure $\mu$. Let $\Gamma \subset[E]$ be a finitely generated group, where $[E]$ is the equivalence relation defined by $\Phi$. So, we have an action $\Phi_{\Gamma}: \Gamma \curvearrowright\{0,1\}^{\mathbf{N}}$. Our goal is to construct a compressed sofic representation of $\Gamma$. Let $\left\{\gamma_{n}\right\}_{n=1}^{\infty}$ be an enumeration of the elements of $\Gamma$. Let $\epsilon_{n}=\mu\left(F i x\left(\Phi_{\Gamma}\left(\gamma_{n}\right)\right) / 2\right.$. Since $\Gamma$ is in the full group, $\epsilon_{n}>0$. Now, fix a subset $F \subseteq \Gamma$ and $\epsilon>0$. We need to construct a map $\Theta: F \rightarrow \operatorname{Map}(A)$ for some finite set $A$ such that if $\gamma_{i}, \gamma_{j}, \gamma_{i} \gamma_{j} \in F$, then

$$
\begin{gathered}
d_{H}\left(\Theta\left(\gamma_{i} \gamma_{j}\right) \Theta\left(\gamma_{i}\right) \Theta\left(\gamma_{j}\right)\right)<\epsilon, \\
d_{H}\left(\Theta\left(\gamma_{i}\right), 1\right)>\epsilon_{i} .
\end{gathered}
$$

Let $\left\{s_{1}, s_{1}^{-1}, s_{2}, s_{2}^{-1}, \ldots, s_{m}, s_{m}^{-1}\right\}$ be a symmetric generating set for $\Gamma$. Observe that we have an action $\Sigma_{\Gamma}: \mathbf{F}_{m} \curvearrowright\{0,1\}^{\mathbf{N}}$ preserving $\mu$ such that $\Sigma_{\Gamma}(\delta)=$ $\Phi_{\Gamma}(\tau(\delta))$, where $\tau: \mathbf{F}_{m} \rightarrow \Gamma$ is the natural quotient map. A dyadic $E$-map of depth $k$ is a Borel map $Q: X \rightarrow X$ defined in the following way. For each $\rho \in\{0,1\}^{k}$ we pick $w_{Q}(\rho) \in \mathbf{F}_{k} \subset \mathbf{F}_{\infty}$ and define $Q(x)=\Phi\left(w_{Q}(\rho)\right)(x)$ if the first $k$-coordinate of $x$ is $\rho$.

A dyadic approximation of $\Gamma$ is a sequence of families $\left\{Q_{k}\left(s_{i}\right)\right\}_{i=1}^{m},\left\{Q_{k}\left(s_{i}^{-1}\right)\right\}_{i=1}^{m}$, where for any $1 \leq i \leq m$ :

- $Q_{k}\left(s_{i}\right): X \rightarrow X, Q_{n}\left(s_{i}^{-1}\right): X \rightarrow X$ are dyadic $E$-maps of depth $k$.

- $\lim _{k \rightarrow \infty} \mu\left(\left\{x \in X \mid Q_{k}\left(s_{i}\right)(x) \neq \Sigma_{\Gamma}\left(s_{i}\right)(x)\right\}\right)=0$.

- $\lim _{k \rightarrow \infty} \mu\left(\left\{x \in X \mid Q_{k}\left(s_{i}^{-1}\right)(x) \neq \Sigma_{\Gamma}\left(s_{i}\right)(x)\right\}\right)=0$.

We do not require $Q_{k}$ to be a bijection. Nevertheless, $Q_{k}$ can be extended to a homomorphism from $\mathbf{F}_{m}$ to $\operatorname{Map}(X)$. Note that for simplicity we identified the generating set of $\mathbf{F}_{m}$ by the set $\left\{s_{1}, s_{1}^{-1}, s_{2}, s_{2}^{-1}, \ldots, s_{m}, s_{m}^{-1}\right\}$.

Since all the $\Sigma_{\Gamma}\left(s_{i}\right)$ 's are Borel bijections, such dyadic approximations clearly exist. The following lemma is an immediate consequence of the definition of the dyadic approximation.

Lemma 3.1. For any $\delta \in \mathbf{F}_{m}$

$$
\lim _{k \rightarrow \infty} \mu\left(F i x\left(Q_{k}(\delta)\right)\right)=\mu\left(F i x\left(\Sigma_{\Gamma}(\delta)\right)\right) .
$$

Proposition 3.1. There exists a sequence of mappings $\hat{\Theta}_{k}: \mathbf{F}_{m} \rightarrow \operatorname{Map}\left(B_{k}\right)$, where $\left|B_{k}\right|<\infty$ such that for any $\delta \in \mathbf{F}_{m}$,

$$
\left.\lim _{k \rightarrow \infty}\left(\mu\left(F i x\left(Q_{k}(\delta)\right)\right)\right)-\frac{\left|F i x\left(\hat{\Theta}_{k}(\delta)\right)\right|}{\left|B_{k}\right|}\right)=0 .
$$

That is,

$$
\lim _{k \rightarrow \infty} \frac{\left|F i x\left(\hat{\Theta}_{k}(\delta)\right)\right|}{\left|B_{k}\right|}=\mu\left(\operatorname{Fix}\left(\Sigma_{\Gamma}(\delta)\right)\right) .
$$

Proof. Let $\Phi_{k}: \mathbf{F}_{k} \curvearrowright\{0,1\}^{\mathbf{N}}$ be the restriction of $\Phi$. Since $\Phi$ is sofic, there exists a sequence of mappings $\left\{\iota_{k}^{n}: \mathbf{F}_{k} \curvearrowright \operatorname{Perm}\left(C_{k, n}\right)\right\}_{n=1}^{\infty}$, where $C_{k, n}$ is a finite $\{0,1\}^{k}$-vertex labeled graph such that for any $t \geq 1$ and $\kappa \in U_{k}^{t}$,

$$
\lim _{n \rightarrow \infty} \mu_{\iota_{k}^{n}}^{t, k}(\kappa)=\mu_{\Phi_{k}}^{t}(\kappa) \text {. }
$$


Recall that $Q_{k}$ is not necessarily an action, only a homomorphism from $\mathbf{F}_{m}$ to $\operatorname{Map}(X)$. Hence, the local statistics of $Q_{k}$ cannot be described using the elements of $U_{k}^{t}$ as in the case of honest $\mathbf{F}_{m}$-actions. So, let $W_{k}^{t}$ be the set of isomorphism classes of rooted $t$-balls of vertex degrees at most $2 m$, where the vertices are labeled by elements of the set $\{0,1\}^{k}$ and the edges (possibly loops) are labeled by subsets of $\left\{s_{1}, s_{1}^{-1}, s_{2}, s_{2}^{-1}, \ldots, s_{m}, s_{m}^{-1}\right\}$. Note that $U_{k}^{t} \subset W_{k}^{t}$. Let $x, y \in X$ be points such that $B_{k^{2}}^{\Phi_{k}}(x)$ and $B_{k^{2}}^{\Phi_{k}}(y)$ represent the same element in $U_{k}^{k^{2}}$. Here $B_{k^{2}}^{\Phi_{k}}(x)$ denotes the $k$-ball with respect to the graphing associated to $\Phi_{k}$. Then, by the definition of the dyadic approximations $B_{k}^{Q_{k}}(x)$ and $B_{k}^{Q_{k}}(y)$ represent the same elements in $W_{k}^{k}$. Now we construct a sequence of maps $\hat{\Theta}_{k}^{n}: \mathbf{F}_{m} \curvearrowright \operatorname{Map}\left(C_{k, n}\right)$ the following way:

$$
\hat{\Theta}_{k}^{n}\left(s_{i}\right)(x)=\iota_{k}^{n}\left(w_{Q_{k}\left(s_{i}\right)}(\rho(x))\right)(x),
$$

where $\rho(x)$ is the $\{0,1\}^{k}$-label of $x$. By the previous observation, for any $\delta \in \mathbf{F}_{m}$

$$
\lim _{n \rightarrow \infty} \frac{\left|F i x\left(\hat{\Theta}_{k}^{n}(\delta)\right)\right|}{\left|C_{k, n}\right|}=\mu\left(\operatorname{Fix}\left(Q_{k}(\delta)\right)\right) .
$$

This finishes the proof of the proposition.

Pick a section $\sigma: \Gamma \rightarrow \mathbf{F}_{m}$, that is, a map such that $\tau \sigma=I d$. Let $\hat{\Theta}_{k}$ be as in Proposition 3.1. Define $\Theta_{k}: \Gamma \rightarrow \operatorname{Map}\left(B_{k}\right)$ by

$$
\Theta_{k}(\gamma)=\hat{\Theta}_{k}(\sigma(\gamma))
$$

Then $\left\{\Theta_{k}\right\}_{k=1}^{\infty}$ is a compressed sofic representation of $\Gamma$.

\section{The Proof of Theorem 2}

Let $T: C \rightarrow C$ be a minimal homeomorphism and $\Gamma \subset[[T]]$ be a finitely generated subgroup of the topological full group of $T$ with symmetric generating set $S=\left\{a_{1}, a_{2}, \ldots, a_{k}\right\}$. It is enough to prove that $\Gamma$ is LEF. Let $x \in C$ and consider the $T$-orbit $\left\{T^{n}(x)\right\}_{-\infty}^{\infty}$. We define the map $\phi: \Gamma \rightarrow \operatorname{Perm}(\mathbf{Z})$ of $\Gamma$ into the permutation group of the integers in the following way. Let $\phi(\gamma)(n)=m$, if $\gamma\left(T^{n}(x)\right)=T^{m}(x)$. Since $T$ acts freely on $C, \phi$ is well-defined.

Lemma 4.1. $\phi$ is an injective homomorphism.

Proof. If $\phi(\gamma)=I d$, then $\gamma$ fixes all the elements of the orbit of $x$. Since all the orbits are dense, this implies that $\gamma=1$. The fact that $\phi$ is a homomorphism follows immediately, since $\phi$ is the restriction of the $\Gamma$-action onto the orbit of $x$.

Let $a=\max |n|$, where for some $p \in C$ and $a_{i} \in S, a_{i}(p)=T^{n}(p)$. We define a sequence

$$
l: \mathbf{Z} \rightarrow\{-a,-a+1, \ldots, 0,1, \ldots, a-1, a\}^{S}
$$

in the following way. Let $l(n):=\left(t_{a_{1}}, t_{a_{2}}, \ldots, t_{a_{k}}\right)$, where $a_{i}\left(T^{n}(x)\right)=T^{n+t_{a_{i}}}(x)$. The following lemma is well-known; we prove it for the sake of completeness.

Lemma 4.2. $l$ is a repetitive sequence; that is, if we find a substring $\sigma$ in $l$, then there exists $m \geq 1$ such that for any interval of length $m$ we can find $\sigma$.

Proof. For a point $p \in C$, we can define its $n$-pattern

$$
q_{n}(p):=\{-n,-n+1, \ldots, 0,1, \ldots, n-1, n\} \rightarrow\{-a,-a+1, \ldots, a-1, a\}
$$


by $q_{n}(p)(j):=\left(t_{a_{1}}, t_{a_{2}}, \ldots, t_{a_{k}}\right)$, where $a_{i}\left(T^{j}(x)\right)=T^{j+t_{a_{i}}}(x)$. Observe that the set of points with a given $n$ pattern is closed. Now, let us suppose that for a sequence $\left\{k_{r}\right\}_{r=1}^{\infty} \subset \mathbf{Z}$ the intervals $\left(k_{r}-r, k_{r}+r\right)$ do not contain $\sigma$ as a substring. Then, if $z$ is a limit point of $\left\{T^{k_{r}}(x)\right\}_{r=1}^{\infty}$, no translates of $z$ have $\sigma$ as a part of their $n$-patterns. Therefore the orbit closure of $z$ does not contain $x$, in contradiction with the minimality of $T$.

Now let $r \geq 1$ and consider the string $\sigma_{r}=l_{\mid\{-a r,-a r+1, \ldots, a r-1, a r\}}$, where $a$ is the constant defined above. Note that if $\gamma \in \Gamma$ is the product of at most $r$ generators, then $|\phi(\gamma)(i)-i| \leq$ ar. Pick $n>10 a^{r}$ such that

- $l_{\mid\{-a r+n,-a r+1+n, \ldots, a r-1+n, a r+n\}}=\sigma_{r}$,

- for any $\gamma \in \Gamma$ that is the product of at most $r$ generators, there is $0<j<n$ such that $\gamma(j) \neq j$.

Now we define $\phi_{r}: W^{r} \rightarrow \operatorname{Perm}\left(\mathbf{Z}_{n}\right)$, where $W^{r}$ is the set of elements in $\Gamma$ that are products of at most $r$ generators by $\phi_{r}(i)=\phi(i)(\bmod n)$. Clearly, $\phi_{r}$ is injective, and if $x, y, x y \in W^{r}$, then $\phi_{r}(x) \phi_{r}(y)=\phi_{r}(x y)$. This implies that $\Gamma$ is LEF.

\section{The Proof of Theorem 3}

Let $\alpha: \Gamma^{k} \rightarrow X$ be a transitive and faithful action of the free product group. Consider the Schreier graph $G_{\alpha}$ of the action with respect to the generators of the $k$ cyclic groups $\left\{a_{1}, a_{2}, \ldots, a_{k}\right\}$. Recall that $V\left(G_{\alpha}\right)$ is $X$ and $(x, y) \in E(G)$ if $y=a_{i} x$ for some $i \geq 1$. Hence $G_{\alpha}$ is a connected graph of vertex degree bound $k$.

Proposition 5.1. Let $\alpha$ be as above. Then for any $1 \neq w \in \Gamma^{k}$, there exist infinitely many $y \in X$ such that $\alpha(w)(y) \neq y$.

Proof. We will need the following lemma.

Lemma 5.1. For any finite set $S \subseteq X$, there exists $g \in \Gamma^{k}$ such that $g S \cap S=\emptyset$.

Proof. We define a lazy random walk on $X$ in the following way. For $y \in X$ the transition probability $p(x, y)=l / k$, where $l$ is the number of generators $a_{i}$ such that $a_{i} x=y$. It is well-known (see e.g. [9], 8]) that the probabilities $p_{n}(x, y)$ tend to zero for each pair $x, y \in X$. Now consider the standard random walk on the Cayley graph of $\Gamma^{k}$, the $k$-regular tree. Let $P_{n}(g)$ be the probability being at $g$ after taking $n$ steps starting from the identity. Then,

$$
p_{n}(x, y)=\sum_{g \in \Gamma, g x=y} P_{n}(g) .
$$

By the previous observation, if $n$ is large enough, then

$$
\sum P_{n}(g)<1
$$

where the summation is taken for all $g \in \Gamma^{k}$ such that $g x \in S$, for some $x \in S$. Hence, there exists $g \in \Gamma^{k}$ such that $g S \cap S=\emptyset$.

Now let us suppose that $w \in \Gamma^{k}$ fixes all points of $X$ outside a finite set $S$. That is, $\alpha(w)(S)=S$. Let $g S \cap S=\emptyset$. Then $g w g^{-1}$ fixes all the points of $X$ outside $g S$. Therefore the commutator $\left[w, g w g^{-1}\right]$ fixes all elements of $X$, in contradiction with the assumption that the action is faithful. 
Now fix a vertex $x \in X$ and consider the ball of radius $n, B_{n}(x)$ around $x$. We define an action $\alpha_{n}: \Gamma^{k} \curvearrowright B_{n}(x)$ in the following way. Let $\partial B_{n}(x)$ be the boundary of the ball $B_{n}(x)$, that is, the set of all $y \in B_{n}(x)$ such that there exists $a_{i}$ for which $\alpha\left(a_{i}\right) y \notin B_{n}(x)$. If $y \notin \partial B_{n}(x)$, then let $\alpha_{n}\left(a_{i}\right) y=\alpha\left(a_{i}\right) y$. If $y \in \partial B_{n}(x)$ and $\alpha\left(a_{i}\right) y \notin B_{n}(x)$, then let $\alpha_{n}\left(a_{i}\right)(y)=y$. Finally, if $y \in \partial B_{n}(x)$ and $\alpha\left(a_{i}\right) y \in B_{n}(x)$, then let $\alpha_{n}\left(a_{i}\right)(y)=\alpha\left(a_{i}\right)(y)$. Now let $L_{k}^{n}=\{0,1\}^{B_{n}(x)} \rtimes_{\alpha_{n}} \alpha_{n}\left(\Gamma^{k}\right)$ be the associated finite lamplighter group and $L^{k}=\bigoplus_{x \in X}\{0,1\} \rtimes_{\alpha} \Gamma^{k}$. Our goal is to embed $L^{k}$ into $L_{k}^{n}$ locally. That is, for any finite set $F \subset L^{k}$ we construct an injective map $\Theta: F \rightarrow L_{k}^{n}$ such that $\Theta(f g)=\Theta(f) \Theta(g)$. Recall that each element of $L^{k}$ can be uniquely written in the form $a \cdot w$, where $a \in \bigoplus_{x \in X}\{0,1\}$ and $w \in \Gamma^{k}$. We regard the elements of the lamplighter group as permutations of the set $\bigoplus_{x \in X}\{0,1\}$. If $\kappa \in \bigoplus_{x \in X}\{0,1\}$ and $p \in X$, then

$$
(a \cdot w)(\kappa)_{\mid p}=a(p)+\kappa\left(\alpha\left(w^{-1}\right)(p)\right) .
$$

We will also use the product formula

$$
\left(a_{2} \cdot w_{2}\right)\left(a_{1} \cdot w_{1}\right)=\left(a_{2}+\alpha\left(w_{2}\right)\left(a_{1}\right), w_{2} w_{1}\right),
$$

where $\alpha\left(w_{2}\right)\left(a_{1}\right)(q)=a_{1}\left(\alpha\left(w_{2}^{-1}\right)(q)\right)$. For $l \geq 1$, let $H_{l}$ be the set of elements of $L^{k}$ in the form of $a \cdot w$, where $w$ is a word of length at most $l$ and the support of $a$ is contained in $B_{l}(x)$. For $n \geq l$ we define the map $\tau_{l}^{n}: H_{l} \rightarrow L_{k}^{n}$ by $\tau_{l}^{n}(a \cdot w):=$ $a \cdot \alpha_{n}(w)$.

Lemma 5.2. If $n$ is large enough, then $\tau_{l}^{n}$ is injective.

Proof. If $n$ is large enough, then $B_{n}(x)$ contains a point $y$ such that

- $\alpha(w)(y) \neq y$,

- $d\left(y, \partial B_{n}(x)\right)>l$,

- $d\left(y, B_{l}(x)\right)>l$,

where $d$ is the shortest path distance on the Schreier graph $G_{\alpha}$. Let $\kappa \in \bigoplus_{x \in X}\{0,1\}$ be the element which is 1 at $y$ and zero otherwise. Then

$$
\tau_{l}^{n}(a \cdot w)(\kappa)_{\mid \alpha_{n}(w)(y)}=1,
$$

hence $\tau_{l}^{n}(a \cdot w)$ is not trivial.

The following lemma finishes the proof of Theorem 3

Lemma 5.3. Suppose that $\left(a_{1} \cdot w_{1}\right),\left(a_{2} \cdot w_{2}\right)$ and $\left(a_{2} \cdot w_{2}\right)\left(a_{1} \cdot w_{1}\right) \in H_{l}$ and $n$ is large enough. Then

$$
\tau_{l}^{n}\left(\left(a_{2} \cdot w_{2}\right)\right) \tau_{l}^{n}\left(\left(a_{1} \cdot w_{1}\right)\right)=\tau_{l}^{n}\left(\left(a_{2} \cdot w_{2}\right)\left(a_{1} \cdot w_{1}\right)\right) .
$$

Proof. We need to prove that

$$
\left(a_{2} \cdot \alpha_{n}\left(w_{2}\right)\right)\left(a_{1} \cdot \alpha_{n}\left(w_{1}\right)\right)=\left(a_{2}+\alpha\left(w_{2}\right)\left(a_{1}\right)\right) \cdot \alpha_{n}\left(w_{2} w_{1}\right)
$$

holds in $L_{k}^{n}$. Fix an element $\kappa \in\{0,1\}^{B_{n}(x)}$. Let $n>10 l$ and $\left.d\left(p, \partial B_{n}(x)\right)\right)>5 l$. Then

$$
\left(a_{2} \cdot \alpha_{n}\left(w_{2}\right)\right)\left(a_{1} \cdot \alpha_{n}\left(w_{1}\right)\right)(\kappa)_{\mid p}=\left(a_{2} \cdot w_{2}\right)\left(a_{1} \cdot w_{1}\right)(\bar{\kappa})_{\mid p}
$$

and

$$
\left(a_{2}+\alpha\left(w_{2}\right)\left(a_{1}\right) \cdot \alpha_{n}\left(w_{2} w_{1}\right)\right)(\kappa)_{\mid p}=\left(a_{2}+\alpha\left(w_{2}\right)\left(a_{1}\right) \cdot\left(w_{2} w_{1}\right)\right)(\bar{\kappa})_{\mid p},
$$


where $\bar{\kappa}$ is an extension of $\kappa$ onto $X$. On the other hand, if $\left.d\left(p, \partial B_{n}(x)\right)\right) \leq 5 l$, then

$$
\begin{aligned}
& \left(a_{2} \cdot \alpha_{n}\left(w_{2}\right)\right)\left(a_{1} \cdot \alpha_{n}\left(w_{1}\right)\right)(\kappa)_{\mid p}=\alpha_{n}\left(w_{2}\right) \alpha_{n}\left(w_{1}\right)(\kappa)_{\mid p} \\
& \left.=\alpha_{n}\left(w_{2} w_{1}\right)(\kappa)_{\mid p}=\left(a_{2}+\alpha\left(w_{2}\right)\left(a_{1}\right)\right) \cdot \alpha_{n}\left(w_{2} w_{1}\right)\right)(\kappa)_{\mid p} .
\end{aligned}
$$

\section{ACKNOWLEDGEMENT}

The author thanks Nicolas Monod and Gábor Pete for valuable discussions.

\section{REFERENCES}

[1] Gábor Elek and Gábor Lippner, Sofic equivalence relations, J. Funct. Anal. 258 (2010), no. 5, 1692-1708, DOI 10.1016/j.jfa.2009.10.013. MR2566316 (2011j:37009)

[2] Gábor Elek and Endre Szabó, Hyperlinearity, essentially free actions and $L^{2}$-invariants. The sofic property, Math. Ann. 332 (2005), no. 2, 421-441, DOI 10.1007/s00208-005-0640-8. MR2178069 (2007i:43002)

[3] Gábor Elek and Endre Szabó, On sofic groups, J. Group Theory 9 (2006), no. 2, 161-171, DOI 10.1515/JGT.2006.011. MR2220572(2007a:20037)

[4] R. Grigorchuk and K. Medynets, On algebraic properties of topological full groups (preprint) http://arxiv.org/pdf/1105.0719.pdf

[5] M. Gromov, Endomorphisms of symbolic algebraic varieties, J. Eur. Math. Soc. (JEMS) 1 (1999), no. 2, 109-197, DOI 10.1007/PL00011162. MR1694588(2000f:14003)

[6] Kate Juschenko and Nicolas Monod, Cantor systems, piecewise translations and simple amenable groups, Ann. of Math. (2) 178 (2013), no. 2, 775-787, DOI 10.4007/annals.2013.178.2.7. MR3071509

[7] Alexander S. Kechris and Benjamin D. Miller, Topics in orbit equivalence, Lecture Notes in Mathematics, vol. 1852, Springer-Verlag, Berlin, 2004. MR2095154 (2005f:37010)

[8] Russell Lyons, Asymptotic enumeration of spanning trees, Combin. Probab. Comput. 14 (2005), no. 4, 491-522, DOI 10.1017/S096354830500684X. MR2160416 (2006j:05048)

[9] B. Morris and Yuval Peres, Evolving sets, mixing and heat kernel bounds, Probab. Theory Related Fields 133 (2005), no. 2, 245-266, DOI 10.1007/s00440-005-0434-7. MR2198701 (2007a:60042)

[10] Liviu Păunescu, On sofic actions and equivalence relations, J. Funct. Anal. 261 (2011), no. 9, 2461-2485, DOI 10.1016/j.jfa.2011.06.013. MR2826401 (2012j:46089)

[11] A. M. Vershik and E. I. Gordon, Groups that are locally embeddable in the class of finite groups (Russian), Algebra i Analiz 9 (1997), no. 1, 71-97; English transl., St. Petersburg Math. J. 9 (1998), no. 1, 49-67. MR.1458419 (98f:20025)

[12] Benjamin Weiss, Sofic groups and dynamical systems, Sankhyā Ser. A 62 (2000), no. 3, 350-359. Ergodic theory and harmonic analysis (Mumbai, 1999). MR1803462 (2001j:37022)

Department of Mathematics, Lancaster University, Bailrigg, Lancaster, LA1 4YW, UNITED KINGDOM

E-mail address: g.elek@lancaster.ac.uk 